\title{
Highly Spin-Polarized Field Emissions Induced by Quantum Size Effects in Ultrathin Films of Fe on W(001)
}

\author{
Bin $\mathrm{Li},{ }^{1}$ T. C. Leung, ${ }^{2}$ and C. T. Chan ${ }^{1, *}$ \\ ${ }^{1}$ Physics Department, Hong Kong University of Science and Technology, Clear Water Bay, Hong Kong, China \\ ${ }^{2}$ Department of Physics, National Chung Cheng University, Chia-Yi, Taiwan, Republic of China
}

(Received 21 November 2005; published 21 August 2006)

\begin{abstract}
Spin-polarized field emissions from Fe pseudomorphic ultrathin films on W(001) surfaces are studied by density functional calculations. We found that nearly completely spin-polarized field emission currents can be realized in two and four Fe layers on $\mathrm{W}(001)$ and that these systems have the additional advantages of thermal stability and low work functions. The unusually high spin polarizations of the field emission current is traced to the Fe film's quantum size effects leading to spin-polarized quantum well states and surface resonance states.
\end{abstract}

DOI: 10.1103/PhysRevLett.97.087201

Nanostructured systems have enhanced effects due to the confinement of quantized states, and, for the particular case of ultrathin supported films, many amazing structural and electronic properties have been discovered [1]. We will see that such confinement effects can give interesting and potentially useful phenomena in spin-polarized field emission (FE). Although tungsten is the metal of choice for emitter tips, it does not produce spin-polarized FE currents. We propose adding a thin coating of ferromagnetic material to tungsten to generate highly spin-polarized FE. Because the FE electrons tunneling out of a ferromagnetic maLL10514terial surface are usually not strongly polarized [2-5], this is not a simple proposition as we must consider factors like the work function change, thermal stability, and most importantly, the strength of the polarization of electrons tunneling out of the magnetic layer. In our analysis of the quantum size effects of spin-polarized $\mathrm{FE}$, we found that there exist configurations of $\mathrm{Fe} / \mathrm{W}(001)$ under which all the aforementioned conditions are favorable.

$\mathrm{Fe} / \mathrm{W}$ systems have been investigated by many researchers. Chrobok et al. measured spin-polarized FE currents from different Fe films on W tips and massive Fe tips [4]. They did not report on the structural characterization of the films. Other experiments found that $\mathrm{Fe}$ grows pseudomorphically on $\mathrm{W}(001)$ surfaces in the first few monolayers (ML) [6-9]. In particular, the first two ML form wetting layers that are stable upon annealing up to $700 \mathrm{~K}$ [6]. A recent spin-resolved scanning tunneling microscopy experiment [10] investigated the electronic structure of $\mathrm{Fe}$ pseudomorphic films on W(001) surfaces with two to four layers of coverage.

In this Letter, we use density functional calculations to study the spin-polarized FE from pseudomorphic Fe ultrathin films on $\mathrm{W}(001)$. Our results show that the electron spin polarization (ESP) of the FE current depends strongly on the thickness of the Fe layers. In particular, nearly complete spin polarization can be realized in two and four ML of $\mathrm{Fe} / \mathrm{W}(001)$. These configurations have the
PACS numbers: 75.70.Ak, 71.15.Mb, 79.60.Dp, 79.70.+q

additional advantages of energy stability and low work functions.

Our formulation for the FE current is based on the localdensity approximation (LDA), which has been described elsewhere [11]. The FE current density contributed by a state labeled as $\left(\sigma, n, \vec{k}_{\|}\right)$(spin, band, and $k$ point indices) of the emitter under an external electric field ( $E$-field), $F$, can be written as

$$
J_{\sigma, n, \vec{k}_{\|}}=\sum_{\vec{G}_{\|}}\left|f_{\sigma, n}\left(\vec{k}_{\|}+\vec{G}_{\|}, z_{m}\right)\right|^{2} g\left(\varepsilon_{\sigma, n, \vec{k}_{\|}}, \vec{k}_{\|}+\vec{G}_{\|}, z_{m}\right),
$$

where $f_{\sigma, n}\left(\vec{k}_{\|}+\vec{G}_{\|}, z\right)$ is the plane-wave expansion coefficient of the eigenstate wave function in a slab geometry, $\quad g\left(\varepsilon_{\sigma, n, \vec{k}_{\|}}, \vec{k}_{\|}+\vec{G}_{\|}, z_{m}\right)=\frac{\hbar}{\pi m}\left(\frac{2 m e F}{\hbar^{2}}\right)^{1 / 3} \mid \operatorname{Bi}\left[\left(\frac{2 m e F}{\hbar^{2}}\right)^{1 / 3} \times\right.$ $\left.\left(z_{a}-z_{m}\right)\right]\left.\right|^{-2}$ is interpreted as a tunneling factor related to the Airy function, $\mathrm{Bi}, \varepsilon_{\sigma, n, \vec{k}_{\|}}$is the eigenenergy, and $z_{a}$ is the classical turning point for the linear potential in the vacuum region. There is a range of matching point values $z_{m}$ (see [11]) that can be used, and we use $z_{m}=8 \AA$ away from the Fe film surface. The total energy distribution (TED) of the FE current density with spin $\sigma$ can be written as $J_{\sigma}(E)=\sum_{n, \vec{k}_{\|}} \delta\left(E-\varepsilon_{\sigma, n, \vec{k}_{\|}}\right) \eta\left(\varepsilon_{\sigma, n, \vec{k}_{\|}}\right) J_{\sigma, n, \vec{k}_{\|}}$, where $\delta(E)$ and $\eta\left(\varepsilon_{\sigma, n, \vec{k}_{\|}}\right)$means the $\delta$-function and FermiDirac occupation function ( $T$ is set at $78 \mathrm{~K}$ ) respectively [12]. The ESP value is obtained from $P=\left(J_{\text {total }, \uparrow}-\right.$ $\left.J_{\text {total }, \downarrow}\right) /\left(J_{\text {total }, \uparrow}+J_{\text {total }, \downarrow}\right)$, where $J_{\text {total }, \sigma}=\int J_{\sigma}(E) d E$.

The electronic structure of the emitter under an external $E$-field is determined using the projected augmented wave (PAW) method [13-16] and the Perdew-Burke-Ernzerhof (PBE) form [17] of the generalized gradient approximation (GGA) with a plane-wave cutoff of $335 \mathrm{eV}$. We used a slab geometry consisting of 11 (or more, for convergence tests) layers of $\mathrm{W}$ and up to five epitaxial layers of Fe. In agreement with previous calculations $[18,19]$, we found that the ground state for one ML Fe/W(001) is antiferromagnetic, and thus our spin-polarized FE calculations are 
done for two to five Fe layers, which have ferromagnetic ground states. The external $E$-field is applied in the (001) direction. The supercell has a vacuum thickness of $25 \AA$. All atoms are fully relaxed.

Figure 1, panels (a1)-(a4) show our calculated TED curves of the FE currents from the Fe films on W(001) surface under an $E$-field of $0.2 \mathrm{~V} / \AA$. The most important observation is that the ESP values are close to $-100 \%$ (i.e. FE current is nearly entirely minority-spin) for two and four ML. When we examine the calculated local density of states (LDOS), we found that the Fe surface layer is dominated by the minority-spin LDOS near the Fermi level $\left(E_{F}\right)$. While the dominance of minority-spin LDOS near $E_{F}$ is expected and is consistent with the negative ESP from two to four layers, it cannot explain the nearly $-100 \%$ ESP at two and four layers, nor can it explain the positive ESP at five ML of Fe. This observation highlights the importance of the details of the band structure and the wave function characteristics of the emitter.

The dominance of the minority-spin current at two ML is due to quantum well (QW) states, and to see how that happens, we have to examine the details. Previous studies showed that the FE current is dominated by contributions from the states near the $E_{F}$ and $\bar{\Gamma}$, and that states with $s, p_{z}$ and $d_{z}^{2}$ components contribute more FE current than other states. Consequently, we examine the QW state in the Fe film with $\Delta_{1}$ symmetry at $\bar{\Gamma}$. Figure 2 (a) shows the band

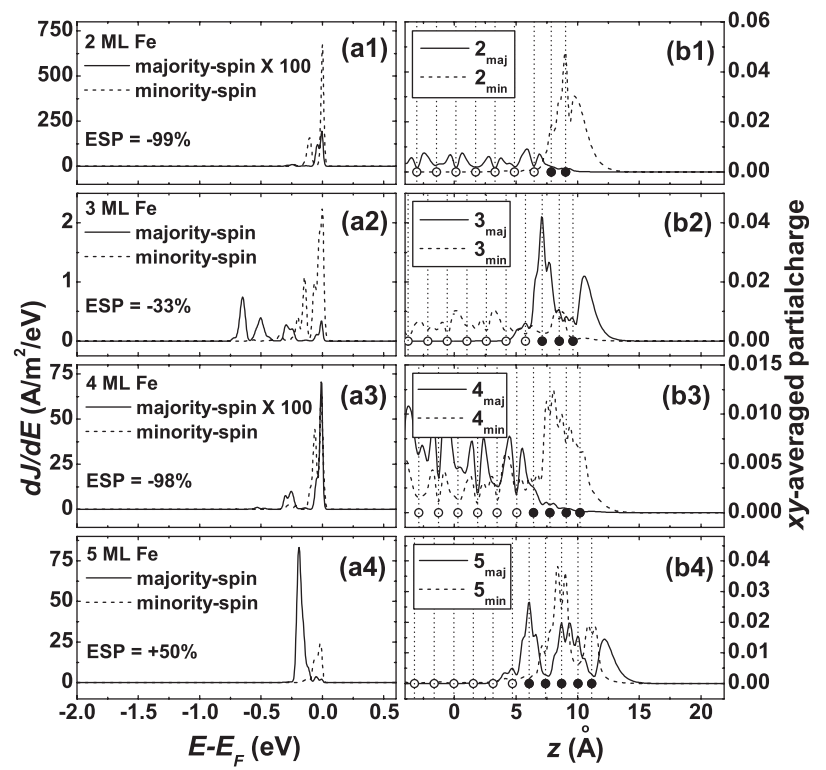

FIG. 1. Panels (a1) through (a4) show the TED curves of the spin-polarized $\mathrm{FE}$ currents from $\mathrm{Fe} / \mathrm{W}(001)$ under $E=$ $0.2 \mathrm{~V} / \AA$, and panels (b1) through (b4) show the $x y$-averaged partial charge distributions of representative states contributing to most of the FE current (solid line: majority-spin; dashed line: minority-spin). The vertical dot lines indicate the atomic positions in the $z$ direction. The solid and open circles mark the $\mathrm{Fe}$ and $\mathrm{W}$ atom positions. structure of a W bcc bulk crystal and Figs. 2(b) and 2(d) show the spin-polarized band structure of an Fe bodycentered tetragonal (bct) bulk crystal (with the same inplane lattice constant as bcc $\mathrm{W}$ and the $z$-coordinates relaxed) along the $\Gamma H$ line. Figs. 2(c) and 2(e) are, respectively, the calculated QW states at $\bar{\Gamma}$ with $\Delta_{1}$ symmetry for the majority and minority spins localized in the Fe film. By comparing Figs. 2(a) and 2(b), we see that there is a wide $\Delta_{1}$ symmetry gap in $\mathrm{W}$ along the (001) direction that allows for the localization of Fe majority-spin QW states, and, indeed, we see from Fig. 2(c) that some majority-spin QW states localized in the Fe film with $\Delta_{1}$ symmetry (at $\bar{\Gamma}$ ) are formed between $-2 \mathrm{eV}$ and $+2 \mathrm{eV}$ relative to the $E_{F}$. For the two and four ML Fe films, all occupied majorityspin QW states are far below $E_{F}$. They therefore have little effect on the ESP of the FE current. For the three and five ML Fe film, there are majority-spin QW states with the number of nodes $(n)$ equal to two and three, respectively, which are closer to $E_{F}$. These states contribute strongly to the majority-spin FE current. The minority-spin QW states are shown in Fig. 2(e). For the two ML Fe films, the minority-spin QW state with $\Delta_{1}$ symmetry and $n=1$ (labeled as 2 min $)$ is at $E_{F}$, while the majority-spin $\mathrm{FE}$ current comes mainly from the $\mathrm{W}$ substrates near $E_{F}$ (a representative state is labeled as $2_{\text {maj }}$ ). The minority-spin (a)

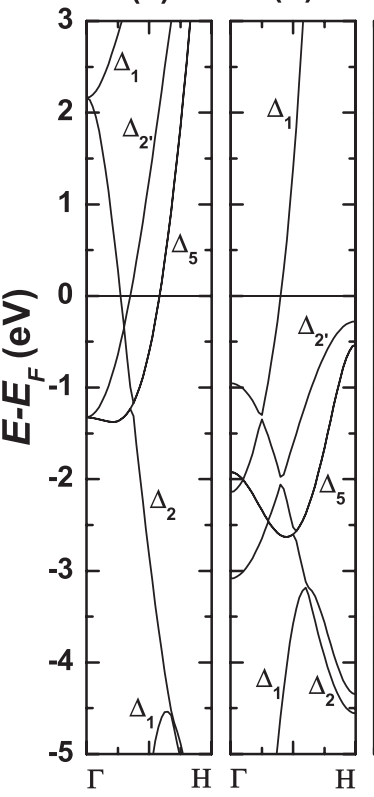

(c)

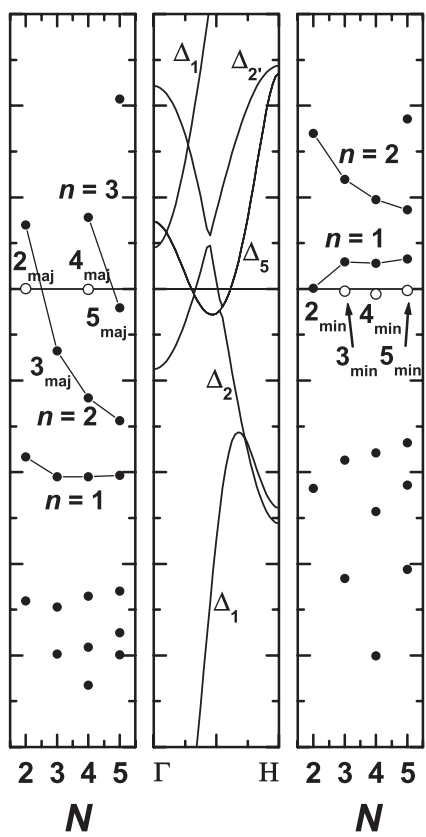

FIG. 2. Bulk energy bands along $\Gamma H$ for bcc W [panel (a)], majority-spin bct Fe [panel (b)] and minority-spin bct Fe [panel (d)]. Panels (c) and (e) show, respectively, majority- and minority-spin QW states (solid dots) at $\bar{\Gamma}$ with $\Delta_{1}$ symmetry for $N$ layers Fe on W(001), and the energies of other non-QW states (open circles) contributing to most of the current are also shown. 
QW state completely dominates the FE current for two reasons. First, the QW state extends further from the surface than the $\mathrm{W}$ substrate state, as can be seen from the (pseudo) charge density along the $z$-direction in Fig. 1, panel (b1). Numerically, we found that the $\mid f_{\sigma, n}\left(\vec{k}_{\|}+\right.$ $\left.\vec{G}_{\|}, z_{m}\right)\left.\right|^{2}$ factor (see Eq. (1)) is much larger for minorityspin $2_{\text {min }}$ state than the $2_{\text {maj }}$ state, and a large $\mid f_{\sigma, n}\left(\vec{k}_{\|}+\right.$ $\left.\vec{G}_{\|}, z_{m}\right)\left.\right|^{2}$ factor gives a large FE current. Second, $2_{\min }$ is of $\Delta_{1}$ symmetry, but the band corresponding to the majorityspin state does not have $\Delta_{1}$ symmetry at $\bar{\Gamma}$.

The minority-spin QW state plays a key role only at two ML. For thicker Fe films, all minority-spin QW states at $\bar{\Gamma}$ point with $\Delta_{1}$ symmetry are far from the $E_{F}$, as shown in Fig. 2(e). For the three ML Fe film, the representative state, $3_{\min }$, which contributes most of the minority-spin current, is off Brillouin zone center and of the extended nature (see Fig. 1, panel (b2)), while the QW state, $3_{\text {maj }}$, is at $\bar{\Gamma}$ and of $\Delta_{1}$ symmetry. These results might suggest that FE should be mostly majority-spin. However, the contrary is found to be true. The FE is again dominated by the minority-spin current, and this is because the $3_{\text {maj }}$ state is at about $0.68 \mathrm{eV}$ below the $E_{F}$, while the $3_{\text {min }}$ state is at $E_{F}$. Thus $3_{\text {maj }}$ have a larger tunneling barrier that inhibits the FE current. When the Fe film becomes thicker, surface resonance states begin to form. At four ML, the majority-spin current is dominated by states near $E_{F}$ that extend into the W bulk [of which a representative state, $4_{\text {maj }}$, is shown in Fig. 1, panel (b3)], but the minority-spin has a surface resonance state $4_{\text {min }}$ mainly localized in the Fe layers just near $E_{F}$ [see Fig. 1, panel (b3)]. This surface resonance state dominates

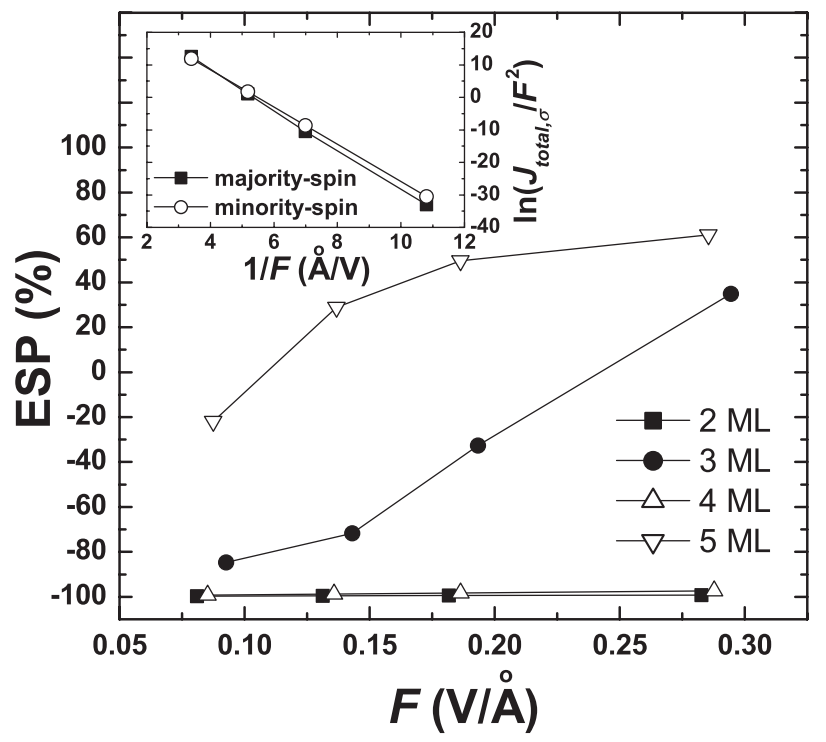

FIG. 3. The ESP of the FE currents from Fe/W(001) under different $E$-field strengths. Inset: the F-N plots of the majorityand minority-spin FE currents (unit: $\mathrm{A} / \mathrm{m}^{2}$ ) from the three $\mathrm{ML}$ $\mathrm{Fe} / \mathrm{W}(001)$. the minority-spin current. The surface resonance state extends much further out of the emitter surface than does the extended state $4_{\text {maj }}$, so the FE current from the four ML $\mathrm{Fe} / \mathrm{W}(001)$ is strongly minority-spin dominant. For the five ML Fe film, the QW state $5_{\text {maj }}$ at the $\bar{\Gamma}$ point and of $\Delta_{1}$ symmetry competes with a surface state, $5_{\min }$, contributing to most of the minority-spin current. In Fig. 1, panel (b4), the partial charge distributions suggest that though these two states are localized in the Fe layers, the $5_{\text {maj }}$ state extends further out of the emitter surface than the $5_{\text {min }}$ state. This leads to a majority-spin-polarized FE current from the five ML Fe film [see Fig. 1, panel (a4)], even though the LDOS at $E_{F}$ is still predominately minorityspin.

The effect of the external $E$-field strength on the spinpolarization of the FE current is shown in Fig. 3. We see that the high minority-spin-polarizations of the FE currents from the two and four ML Fe films are robust up to $F=$ $0.30 \mathrm{~V} / \AA$. On the other hand, the ESP values for the three and five ML Fe films are field dependent. This interesting thickness dependence can be understood qualitatively as follows. According to Fowler-Nordheim (F-N) theory $[20,21]$, the FE current density from a metal surface is $J \propto$ $F^{2} \exp \left\{-[4 \sqrt{2 m} /(3 \hbar e)] \phi^{1.5} / F\right\}$, where $\phi$ is the work function. If the FE current comes mainly from states with energy $\varepsilon_{M}$, the work function $\phi$ should be operationally replaced by an "effective" work function, $\phi_{\text {eff }} \approx$ $\phi+E_{F}-\varepsilon_{M}$ [11]. The F-N plot for the material with a higher 'effective' work function has a larger slope, and thus the current increases more rapidly when the E-field is enhanced. For the three ML Fe film, the majority-spin FE current, $J_{\text {total }, \uparrow}$, has a larger slope in the F-N plot than does

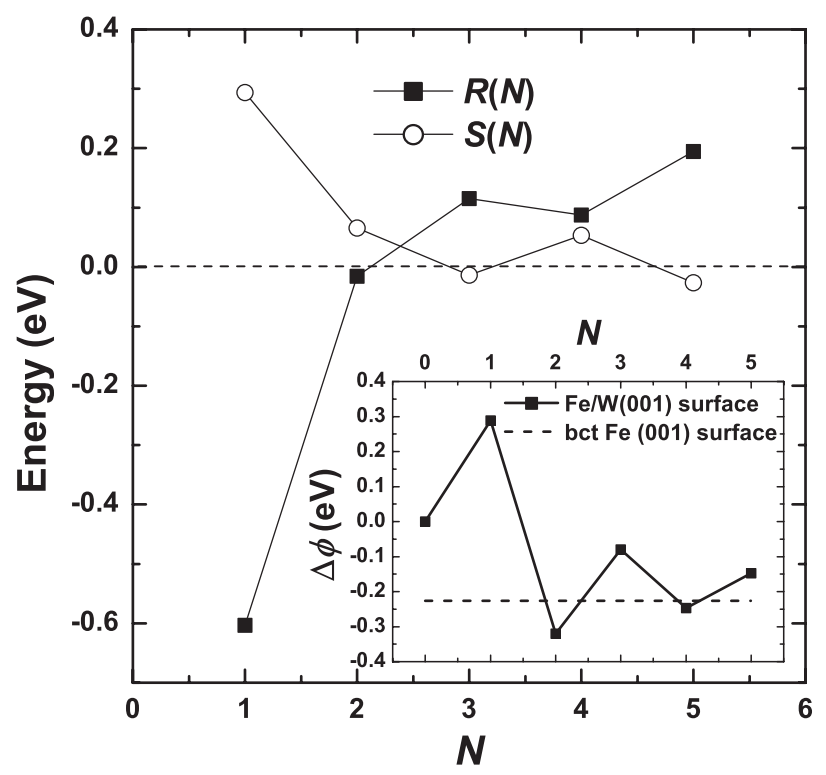

FIG. 4. The functions, $R$ and $S$ (see text) plotted as the functions of the Fe film thickness $N$. Inset: the work functions of the $\mathrm{Fe} / \mathrm{W}(001)$ using the work function of $\mathrm{W}(001)$ as a reference. 
the minority-spin FE current, $J_{\text {total, }}$, because the $3_{\text {maj }}$ state is $0.68 \mathrm{eV}$ lower than the $3_{\text {min }}$ state. The $J_{\text {total }, \uparrow}$ can exceed the $J_{\text {total, } \downarrow}$ when the $E$-field is larger than a threshold, as shown by the inset in Fig. 3. In the case of the five ML Fe film, there is also an energy difference $(\sim 0.20 \mathrm{eV})$ between the $5_{\text {maj }}$ and $5_{\min }$ states.

A good field emitter should be stable and have a low work function. Figure 4 shows the plots of two functions related to the stability of the Fe coating: $R(N)=E(N)-$ $E(N-1)$, and $S(N)=[E(N+1)+E(N-1)] / 2-E(N)$, where $E(N)$ is the surface energy per surface atom for $N$ ML Fe films on W(001). One can see that both $R(1)$ and $R(2)$ are negative, and $R(N)$ turns positive when $N \geq 3$. This means that one and two ML Fe films are thermodynamically stable relative to three-dimensional (3D) bulk Fe islands on $\mathrm{W}(001)$ and one ML of $\mathrm{Fe}$ on $\mathrm{W}(001)$, respectively. This is consistent with the experimental observation that two ML Fe wetting layers are stable upon annealing on $\mathrm{W}(001)$ [6-9], followed by 3D growth for higher coverages beyond $600 \mathrm{~K}[8,9,22]$. The value of $S(4)$ is found to be positive, which suggests that the four ML is metastable (more stable than three and five ML films), consistent with a recent experimental observation [10]. The inset in Fig. 4 gives $\Delta \phi=\phi_{\mathrm{Fe} / \mathrm{W}(001)}-\phi_{\mathrm{W}(001)}$, which is the work function of these $\mathrm{Fe} / \mathrm{W}(001)$ surfaces using $\mathrm{W}(001)$ as a reference. The work function approaches $\phi^{\prime} \mathrm{Fe}(001)^{\prime}$ in an oscillatory manner, where $\phi^{\prime} \mathrm{Fe}(001)^{\prime}$ is the work function of a bct $\mathrm{Fe}(001)$ surface with the in-plane lattice constants matching those of the $\mathrm{W}(001)$ and the $z$-coordinates relaxed. We note, in particular, that the two and four ML Fe covered surfaces have low work functions and thus are favorable to field emissions.

In summary, we studied the spin-polarized FE from the Fe pseudomorphical films on W(001) and identified some configurations that can give strongly spin-polarized FE, and these configurations have the additional advantage of being stable with low work functions. The thicknessdependent FE properties are traced to spin-polarized QW and surface resonance states localized in the Fe layers.

This work was supported by RGC Hong Kong through Grant No. HKUST6152/01P. T.C.L. is supported by National Science Council Grant No. NSC94-2112-M-
194-017, No. NSC94-2120-M-002-005, and a grant of computer time at the National Center for HighPerformance Computing.

*Electronic address: phchan@ust.hk

[1] See, e.g. D.-A. Luh, T. Miller, J. J. Paggel, M. Y. Chou, and T.-C. Chiang, Science 292, 1131 (2001); Zhenyu Zhang, Qian Niu, and Chih-Kang Shih, Phys. Rev. Lett. 80, 5381 (1998).

[2] W. Gleich, G. Regenfus, and R. Sizmann, Phys. Rev. Lett. 27, 1066 (1971).

[3] M. Landolt and M. Campagna, Phys. Rev. Lett. 38, 663 (1977).

[4] G. Chrobok et al., Phys. Rev. B 15, 429 (1977).

[5] M. Landolt and Y. Yafet, Phys. Rev. Lett. 40, 1401 (1978).

[6] P. J. Berlowitz, J. W. He, and D. W. Goodman, Surf. Sci. 231, 315 (1990).

[7] R. L. Fink et al., J. Appl. Phys. 69, 4986 (1991).

[8] H. J. Elmers and J. Hauschild, Surf. Sci. 320, 134 (1994).

[9] W. Wulfhekel et al., Europhys. Lett. 49, 651 (2000).

[10] K. von Bergmann, M. Bode, and R. Wiesendanger, Phys. Rev. B 70, 174455 (2004).

[11] S. F. Huang et al., Phys. Rev. B 72, 035449 (2005).

[12] The currents are calculated on a $20 \times 20 k$-point grid and the $\delta$-function is operationally replaced by Gaussian functions of width ranging from 0.2 to $0.02 \mathrm{eV}$. The ESP values quoted correspond to results extrapolated to zero Gaussian width, so that the smearing near Fermi energy comes from the Fermi function.

[13] G. Kresse and J. Hafner, Phys. Rev. B 47, 558 (1993).

[14] G. Kresse and J. Hafner, Phys. Rev. B 49, 14251 (1994).

[15] G. Kresse and J. Furthmller, Comput. Mater. Sci. 6, 15 (1996).

[16] G. Kresse and J. Joubert, Phys. Rev. B 59, 1758 (1999).

[17] J. P. Perdew, K. Burke, and M. Ernzerhof, Phys. Rev. Lett. 77, 3865 (1996).

[18] J. Chen and J. L. Erskine, Phys. Rev. Lett. 68, 1212 (1992).

[19] R. Q. Wu and A. J. Freeman, Phys. Rev. B 45, 7532 (1992).

[20] R. H. Fowler and L. Nordheim, Proc. R. Soc. A 119, 173 (1928).

[21] See, e.g., C. D. Ehrlich and E. W. Plummer, Phys. Rev. B 18, 3767 (1978).

[22] E. Bauer, Appl. Surf. Sci. 11-12, 479 (1982). 